\title{
A mathematical model for glucose oxidase kinetics, including inhibitory, deactivant and diffusional effects, and their interactions.
}

Jesús Mirón ${ }^{1}, \mathrm{M}^{\mathrm{a}}$ Pilar González ${ }^{1}$, José A. Vázquez ${ }^{1}$ Lorenzo Pastrana $^{2}$, M. A. Murado ${ }^{1 *}$.

${ }^{1}$ Instituto de Investigacións Mariñas (CSIC)

r/ Eduardo Cabello, 6. Vigo-36208. Galicia (Spain)

${ }^{2}$ Área de Bioquímica e Bioloxía Molecular. Facultade de Ciencias de Ourense, Universidade de Vigo. As Lagoas s/n. Ourense-32004, Galicia (Spain)

*Corresponding author: M.A. Murado

Running title: Inhibitory, deactivant and diffusional effects in glucose oxidase kinetics.

Keywords: Glucose oxidase kinetics; inhibitory, deactivant, diffusional effects

\begin{abstract}
Glucose oxidase (GOD) kinetics comprises a group of inhibitory, deactivant and diffusional phenomena interacting with each other in a complex way. These phenomena have given rise to differing interpretations, and their description is hindered by studies at moderate glucose concentrations (usual condition in many applications of the enzyme), the presence (or absence) of catalase in the enzymatic preparation, and the biases associated to the frequent use of linear transformations for kinetic characterizations. The objective of this work is to achieve a kinetic model that integrates all the mentioned effects and allows satisfactory descriptions in conditions (frequent in microbiological context) of wide intervals of substrate concentration, at long reaction periods, and in presence of catalase.
\end{abstract}




\section{INTRODUCTION}

In glucose oxidase (GOD) kinetics, especially when one works on a wide range of substrate concentrations, it is possible to detect a complex group of inhibitory effects due to the substrate (glucose) and product (gluconic acid), that have received little attention.

Substrate inhibition (SI) has been described by Nicol and Duke [1] for the free enzyme from Aspergillus niger, although only at oxygen concentrations lower than $2 \%$ relative to saturation $\left(2 \times 10^{-5} \mathrm{M}\right)$. The conclusion of these observations, cited in the review by Barker and Shirley [2], was confirmed by Tse and Gough [3], who specified that the effect is weak with the reduced form of the enzyme, and also by Kozhukharova et al. [4], working with inmobilized GOD and glucose concentrations above $150 \mathrm{~g} / \mathrm{L}$. Much more numerous, however, are the studies which, in diverse conditions of operation, have either found no evidence of SI [5-9], or avoided the allusion to the phenomenon when describing the kinetic properties of the enzyme [10-14], or provided kinetic constants without including the corresponding to this effect [15-19].

On the other hand, the structural similarity between glucose and gluconic acid allows to suspect the possibility of competitive product inhibition. Nevertheless, the allusions to such mechanism, as well as the work especifically directed to verify it, are scarce. With a correct experimental approach, although nowadays deemed unconventional, Nakamura and Ogura [20] concluded that inhibition by gluconolactone (precursor of gluconic acid), was due to gluconic acid binding with the active site of the enzyme (competitive inhibition). Rogers and Brandt [21] described the same effect with glucal (analogue of glucose with a double bond between carbon 1 and 2). Additionally, although the phenomenon is not directly transferable to enzymologic domain, it is worth noting that Velizarov and Beschkov [22] found substrate and product inhibition in the conversion of glucose to gluconic acid in cultures of Gluconobacter oxydans. Nevertheless, neither the review of Barker and Shirley [2], nor the most recent one of Crueger 
and Crueger [23] include gluconic acid among the GOD inhibitors, and Tse et al. [24], working with GOD and immobilised catalase on synthetic membranes, do not corroborate this effect.

These discrepancies could be due to structural differences in enzymes of different origin, such as those noted by several authors $[6,13,25,26]$. However, other causes may also play a role, for example, moderate glucose concentrations, which are usual conditions in many applications of GOD. Another may be the frequent use of linear transformations (e.g. Lineweaver-Burk) for the kinetic description. This approach is very sensitive to experimental error and the cause of substantial biases, particularly when applied to cases with substrate inhibition. Finally, the frequent presence of catalase in the preparations of GOD tends to obscure a kinetic profile which, although due to partial inactivation of the enzyme by the hydrogen peroxide co-product of the reaction, contributes to focus the attention towards the possibility of inhibitory effects.

The aim of this work is to study in more detail these phenomena, and propose corresponding descriptive models.

\section{MATERIALS AND METHODS}

\section{1: Enzymes employed}

Two preparations of GOD (EC 1.1.3.4) were studied, both from A. niger. The first was the product SIGMA G-7141 (limits of activity: 15,000-25,000 units per gram of solid, without added oxygen. Unit definition: required amount of enzyme to oxidize $1 \mu$ mole of $\beta$-D-glucose to Dgluconolactone and $\mathrm{H}_{2} \mathrm{O}_{2}$ per minute, at $35^{\circ} \mathrm{C}, \mathrm{pH}=5.1$ ). The second was obtained from a submerged culture of $A$. niger CBS 554-65 [27] by the following procedure. When the maximum of 
GOD activity was reached, the filtered medium (50 L) was concentrated by membrane ultrafiltration $(100 \mathrm{kDa})$ to a final volume of $2.5 \mathrm{~L}$. The retentate was washed by diafiltration, continuously adding water (up to $\sim 10 \mathrm{~L}$ ) at an equivalent rate to that permeate outflowing. Ultrafiltration was continued to make the washed retentate up to $\sim 0.4 \mathrm{~L}$ and this final volume was freeze dried to obtain a dry powder. Table 1 shows the main characteristics of both preparations.

The catalase (EC 1.11.1.6) used, as an additive and as a standard to determine its presence in the GOD preparations, was the product SIGMA C-3515. Limits of activity: 4,000-8,000 units per mg protein. Unit definition: required amount of enzyme to descompose $1 \mu$ mole of $\mathrm{H}_{2} \mathrm{O}_{2}$ per min at $25^{\circ} \mathrm{C}, \mathrm{pH}=7.0$, while the $\mathrm{H}_{2} \mathrm{O}_{2}$ concentration (followed by means of the decrease in absorbance at $240 \mathrm{~nm}$ ) falls from 10.3 to $9.2 \mathrm{mM}$.

\section{2: Analytical methods}

All reagents used were of analytical quality (SIGMA).

GOD and hydrogen peroxide: In the method of Lloyd and Whelan [28] for the determination of GOD, the reaction catalysed by this enzyme is linked to the decomposition of hydrogen peroxide with peroxidase (PO) and oxidation of the chromogenic reagent $O$ dianisidine.

$$
\begin{aligned}
& \text { Glucose }+\mathrm{O}_{2} \stackrel{\text { GOD }}{\longrightarrow} \text { Gluconolactone } \leftrightarrow \text { Gluconate }+\mathrm{H}_{2} \mathrm{O}_{2} \\
& \mathrm{H}_{2} \mathrm{O}_{2}+\text { o-dianisidine } \stackrel{\text { Po }}{\longrightarrow} \text { o-dianisidine (ox) }+\mathrm{H}_{2} \mathrm{O}
\end{aligned}
$$

This sequence of reactions is applicable to several objectives, among them: (a) to quantify the enzymatic activity of a sample with an unknown amount of GOD, (b) to quantify, in a typical Michaelian approach, the amount of hydrogen peroxide (and, consequently, gluconate) that produces a known activity of GOD in the presence of variable concentrations of glucose. 
In both cases, analysis was performed according to the method of Fiedurek et al. [29]. In the basic method for determination of GOD, the reagents used were: $\boldsymbol{A}$ : glucose, $18 \mathrm{mg} / \mathrm{mL}$; peroxidase, $16 \mathrm{EU} / \mathrm{mL}$, and glycerol, $0.53 \mathrm{~g} / \mathrm{mL}$, in $0.2 \mathrm{M}$ citric-phosphate buffer, $\mathrm{pH}=5.1$. $\boldsymbol{B}:$ : dianisidine, $2 \mathrm{mg} / \mathrm{mL}$ in distilled water. $C$ : sample with an unknown amount of GOD. The reaction was carried out by mixing $1.9 \mathrm{~mL}$ of $\boldsymbol{A}$ and $0.1 \mathrm{~mL}$ of $\boldsymbol{B}$, maintaining the mixture for 10 minutes at $30^{\circ} \mathrm{C}$, and then adding $0.1 \mathrm{~mL}$ of $\boldsymbol{C}$. After shaking for 15 minutes at $200 \mathrm{rpm}$ and $30^{\circ} \mathrm{C}$, the reaction was stopped by adding $3 \mathrm{~mL}$ of $5 \mathrm{~N} \mathrm{HCl}$ and absorbance was measured at 525 nm (oxidised o-dianisidine). As a standard a series of dilutions of GOD SIGMA G-7141 was used (a suitable interval is $0.1-1.0 \mathrm{EU} / \mathrm{mL}$ ) in place of solution $\boldsymbol{C}$.

This method permits a variation (recommended by SIGMA) which consists of eliminating the glycerol from solution $\boldsymbol{A}$, making the reaction mixture in a spectrophotometric cell and measuring absorbance at $435 \mathrm{~nm}$ every 30 seconds over a period of 5 minutes maximum, taking as the reaction rate the slope of the stretch in which the variation of the absorbance is linear.

Although both procedures provide equivalent results, the first was preferred in the Michaelian experiments. The main reason was the need to work under controlled agitation conditions, an important factor when it became necessary to study the possible diffusional restrictions due to gluconate. In this case, the basic method was applied with the following variations: 1) In solution $\boldsymbol{A}$ the glycerol was eliminated, the concentration of peroxidase was increased to $300 \mathrm{EU} / \mathrm{ml}$, the concentration of glucose was varied within the desired range and, when the effects of gluconate were studied, the desired concentrations of the acid were added. 2) The reaction time was reduced to 4 minutes. 3) The absorbance was measured at $525 \mathrm{~nm}$. 4) As a standard a series of dilutions of $\mathrm{H}_{2} \mathrm{O}_{2}$ was used (a suitable interval is $0.5-5.0 \mathrm{mM}$ ) in place of solution $C$.

One alternative which was also assayed in the Michaelian approach was the determination of gluconate formed. To do this, the reaction was stopped with $4 \mathrm{~mL}$ of $1 \mathrm{~N} \mathrm{HCl}$ and, after 2 
hours, the solution was taken to $\mathrm{pH}=10-11$ with $2 \mathrm{~N} \mathrm{KOH}$, and gluconate determined by means of the method described below. The results did not show significant differences from those of the $o$-dianisidine reaction which, simpler and more economical, was the method finally adopted. For long reaction periods $(\sim 75 \mathrm{~h})$ the necessary sterile conditions were achieved by filtering all solutions through a $0.22 \mu \mathrm{m}$ membrane (the usual method of sodium azide creates interferences with the reaction catalysed by the GOD).

Catalase: The method proposed by SIGMA was followed: $100 \mu \mathrm{L}$ of the enzyme solution was mixed in a $1 \mathrm{~cm}$ cell with $2.9 \mathrm{~mL}$ of a substrate solution $\left(100 \mu \mathrm{L} 30 \% \mathrm{H}_{2} \mathrm{O}_{2}\right.$ in $50 \mathrm{~mL}$ phosphate of buffer $0.05 \mathrm{M} ; \mathrm{pH}=7.0$ ). The disappearance of $\mathrm{H}_{2} \mathrm{O}_{2}$ was measured as the decrease in absorbance at $240 \mathrm{~nm}$. Under these conditions (initial value $>0.450$ ) the reproducibility is good in the period of time required for the absorbance to decrease between 0.450 and 0.400 , which corresponds to the decomposition of $3.45 \mu \mathrm{M} \mathrm{H}_{2} \mathrm{O}_{2}$. Accordingly, the concentration of catalase in the sample was $3.45 /($ minutes $\times 0.1) \mathrm{EU} / \mathrm{mL}$, where $1 \mathrm{EU}$ is the quantity of catalase which decomposes $1 \mu \mathrm{M} \mathrm{H} \mathrm{H}_{2} \mathrm{O}_{2} \min ^{-1}$.

Glucose: 4-aminoantipyrine method [30].

Gluconic acid: specific method of Möllering and Bergmeyer [31] based on the measurement of $\mathrm{NADPH}+\mathrm{H}^{+}$formed in the process:

D-gluconate + ATP + Gluconate kinase $\rightarrow$ D-gluconate-6-P + ADP

D-gluconate-6-P $+\mathrm{NADP}^{+}+6-\mathrm{P}-$ Gluconate dehydrogenase $\rightarrow$ Ribulose-5-P $+\mathrm{NADPH}^{+}+\mathrm{H}^{+}+\mathrm{CO}_{2}$

Gluconolactone potentially present is quantitatively converted to gluconate by taking the sample $\mathrm{pH}$ to $10-11$ with addition of $\mathrm{KOH} 2 \mathrm{~N}$, and maintaining this $\mathrm{pH}$ for 15 minutes. Thereafter, prior to analysis, the $\mathrm{pH}$ was adjusted to $7-8$ with $0.1 \mathrm{~N} \mathrm{HCl}$.

\section{3: Other methods}


The initial equations of kinetic descriptions are shown in Table 2. To avoid the biases associated with linearisation procedures, the calculation of the coefficients was performed with non-linear methods. The quadratic differences between experimentally determined and modelpredicted values were minimised (quasi-Newton). The experimental plan for description of the interactions between GOD and catalase will be described below.

\section{RESULTS AND DISCUSSION}

\section{1: Preliminary results}

In Figure 1 the results of two conventional Michaelian experiments with the two GOD preparations (Table 1) are compared at a concentration of $1 \mathrm{EU} / \mathrm{mL}$ in the incubation mixture and substrate concentration between 1 and $100 \mathrm{~g} / \mathrm{L}$. In both cases, the fits (quasi-Newton) to equations (1) and (2) of Table 2 support the hypothesis of SI existence, which is most prominent in the purest preparation.

The isoelectric focusing of both preparations showed no differences in the 4 bands of GOD, and supplementation of the purest preparation with FAD until the FAD/GOD ratio of the least pure preparation did not change its activity. Therefore, the ratio catalase/GOD was suspected to be involved in the apparent kinetic differences. It should be noted that hydrogen peroxide, with deactivating action on GOD, is a co-product of the enzymatic reaction, and therefore the high substrate concentrations imply high rates of peroxide production. Accordingly, it may be assumed that certain combinations of kinetic parameters cause a decrease in the effective GOD from a certain substrate level, producing profiles showing a rate decrease from a maximum. The situation can be schematically simulated on the basis of the following assumptions: 
The molar rates of formation of the products (gluconic acid: $v G n H$ and hydrogen peroxide: $\vee O X)$ are the same and can be modeled by means of the Michaelis-Menten equation at each time instant. Where $S$ and $G O D$ are the concentrations of substrate and enzyme:

$$
v G n H=v O X=\frac{V_{m} S}{K_{m}+S} G O D
$$

$O X$ formed after a time interval $t$ is obtained by numerical integration of (6) with respect to time:

$$
O X=\sum_{t=0}^{t}\left[\frac{V_{m} S}{K_{m}+S} G O D\right]
$$

Accepting as the simplest kinetic hypothesis that peroxide deactivates the enzyme in a second order reaction (first order in each of the species, kinetic rate constant $=g$ ), the rate of deactivation of GOD in each time instant will be:

$$
u G O D=g O X G O D
$$

and the active enzyme after a time interval $t$, where $G O D_{0}$ is the initial concentrations, is:

$$
G O D=G O D_{0}-\sum_{\mathrm{t}=0}^{t}(g O X \quad G O D)
$$

Figure 2 shows a simulation of this type of system, using the equations (6) to (9) with arbitrary parameters (specified in the figure) for initial concentrations and coefficients. Although a more realistic model ought to include other phenomena as will be seen later, the schematism of such a simulation does not affect the conclusions of interest here: 1) in a typical Michaelian 
experiment, the effect of the hydrogen peroxide can produce a decreasing rate from a maximum value, with superficially similar profiles to those due to SI. However: 2) the fall from a maximum only occurs when working at rates far from the initial rate, and 3) the fit of those profiles to equation (2) of Table 2 (true substrate inhibition) is poor and presents clear autocorrelation in the residuals.

Accordingly, the discrimination between the effects of SI and hydrogen peroxide tend to become more problematic as the reaction period increases and, naturally, as the experimental error increases. These factors therefore acquire more importance than usual in this type of test. In fact, comparing the proportions of product formed and activity of GOD remaining in buffered media, at long reaction periods (Figure 3) the apparent stability of the most impure preparation is greater, and addition of a catalase supplement equates the results of both preparations. In a Michaelian approach, however, the impure supplemented preparation reproduces the kinetic profile of Figure 1, without contributing to the disappearance of the effect attributed to SI.

\section{2: Substrate inhibition}

Considering the fact that the presence of catalase eliminates the interference of hydrogen peroxide in the evaluation of SI, it was decided to substantiate the extent of this effect with the most impure preparation in a new Michaelian experiment. The conditions were the same as previously, but increasing the domain of the substrate concentration to $250 \mathrm{~g} / \mathrm{L}$. The experiment was carried out by triplicate, and confidence intervals $(\alpha<0.05)$ are represented in Figure 4. Again, the fit of the values (quasi-Newton, parameters in Table 3) to equations (1) and (2) of Table 2 suggested SI. The revised conclusion, now with no possible interference by peroxide, must consider the following factors. 
As Tan et al. [32] have shown in terms applicable to this work, equation (2) of Table 2 can be derived from the existence in the enzyme of specific points, different from the active site, whose binding to the substrate determines inhibition. This may justify the discrepancies alluded to in the introduction in terms of the differences between enzymes of different origin. However, equation (2) also adequately describes the phenomena derived from diffusion restrictions in the reaction mixture. The effect is clear with polymeric substrates, whereby the effect increases with the degree of polymerisation [33, 34], and generates kinetics formally indistinguishable from SI [35]. Accordingly, and in absence of evidence for the existence of specific points of SI, the results in Figure 4 could translate this second type of phenomena, which are probably more independent of the enzyme origin. Although for GOD it is unreasonable to attribute significant diffusional effects to the substrate, the high viscosity of gluconic acid could make a contribution, in addition to its possible role as a competitive inhibitor.

\section{3: Product inhibition}

Product inhibition was studied by comparing the rates of a control under the previous conditions (substrate up to $150 \mathrm{~g} / \mathrm{L}$ ), with those obtained with $2.5 ; 5 ; 10$ and $20 \mathrm{~g} / \mathrm{L}$ gluconic acid $(12.75 ; 25.49 ; 50.98$ and $101.96 \mathrm{mM})$. Figure 5 shows the resulting fits to the models discussed below.

With the most moderate levels of substrate tested here, the characteristic profile of SI in the control without initial gluconic acid was less prominent, despite the fact that the best fits were again obtained with equation (2) of Table 2 . Therefore, this was taken as the starting point in which other possible inhibitory effects were introduced: 
Competitive inhibition: $\quad V=\frac{V_{m} S}{K_{m}\left(1+K_{c} I_{c}\right)+S\left(1+K_{s} S\right)}$

Uncompetitive inhibition: $\quad V=\frac{V_{m} S}{K_{m}+S\left(1+K_{s} S\right)\left(1+K_{a} I_{a}\right)}$

Non-competitive inhibition: $\quad V=\frac{V_{m} S}{\left[K_{m}+S\left(1+K_{s} S\right)\right]\left(1+K_{n} I_{n}\right)}$

Since under these conditions the classic methods of linearisation were inadequate, the evaluation criterion was based on the ability of the proposed models to simultaneously solve (quasi-Newton) the five series of results.

A superficial examination of figure 5 suggests that inhibition by gluconic is not, or not only, competitive, since it produces a decrease in the maximum rate. In fact, the fit to equation (10) is poor: at high levels of gluconic acid the rate is overestimated, and low levels create SI as an artefact. It may be concluded, therefore, that the effect of gluconic acid is more important than proposed by the model, whereby the fitting method (in absence of the adequate term) put too much emphasis on the coefficient $K_{s}$. Additionally, models (11) and (12) were unsatisfactory, although the latter produced profiles between observed and expected results somewhat more approximated and correlations close to linearity $(r=0.982$; figure 6A).

Accordingly, the noncompetitive model can be accepted as a base for improvement with additional assumptions, guided by the analysis of the residuals generated by the corresponding equations. Such residuals demonstrate a curvature in the noncompetitive model which suggests the absence of a second order term (Figure 6A). Therefore, in this case the noncompetitive term can be transformed from $\left(1+K_{n} I\right)$ to $\left(1+K_{n} I^{2}\right)$ : 


$$
V=\frac{V_{m} S}{\left[K_{m}+S\left(1+K_{s} S\right)\right]\left(1+K_{n} I_{n}^{2}\right)}
$$

The modification raises the randomness of the distribution of the residuals, reduces the curvature of the corresponding function (Figure 6B) and improves the correlation between observed and expected values $(r=0.993)$. However, the criterion for introducing this modification must also validate competitive mechanism (Figure $6 \mathrm{C} ; r=0.997$ ) which, alone incapable of explaining the results, is now the only assumption which improves the fit of the equation (13). Table 4 resumes the characteristics of the equations discussed, leading to the proposition of the following descriptive model:

$$
V=\frac{V_{m} S}{\left[K_{m}\left(1+K_{c} I_{c}\right)+S\left(1+K_{s} S\right)\right]\left(1+K_{n} I_{n}^{2}\right)}
$$

Although competitive inhibition of GOD by gluconic acid is not problematic, the justification of the qualified term of "second order noncompetitive effect" seems more obscure. Accordingly, it should be noted that it is difficult to find an alternative to the equation (14), with other reasonable solutions being very far from a satisfactory fit. In fact, the hypothesis of any two or three simultaneous inhibitory effects generates unacceptable residuals and biases. An additional uncompetitive term in (14) produces a negative $K_{a}$ coefficient, and the calculation with the restriction $K_{a} \geq 0$ reproduces (14) with $K_{a}=0$. Finally, if the concentration of gluconic acid is considered as the sum of the initial plus the average concentration produced during the incubation, again one obtains (14), with variations lower than $10^{-5}$ in $K_{n}$ and $K_{c}$.

To suggest an underlying physical phenomenon of the quadratic term in (14), a direct interpretation of the expression would suggest that there is a retarding effect of enzyme-substrate binding. Moreover, the effect appears not to involve blocking of the active site and is of greater 
intensity (thus second order) than a conventional non-competitive inhibitor. Furthermore, this phenomenon may also be related to the cited restrictions on diffusion due to gluconic acid viscosity, and in fact, the effect decreases when increasing the stirring rate of the reaction mixture, a factor, however, that is not possible to include directly in a michaelian model. Thus, it can be speculated that the viscosity retards the enzyme-substrate binding and the enzymeproduct separation, by mechanisms not strictly agreeable to those of non-competitive mechanism or SI. Therefore, they cannot be described adequately with these functional forms.

\section{4: Effect of catalase. A dynamic model}

To study the effect of catalase on GOD activity, two series of tests were performed with GOD $(2 \mathrm{EU} / \mathrm{mL})$ and glucose $(150 \mathrm{mg} / \mathrm{mL})$ in the absence and presence of $100 \mathrm{EU} / \mathrm{mL}$ catalase. The incubation period was prolonged for 70 hours at $30^{\circ} \mathrm{C}$. The experimental results, fit to the model discussed below, are shown in Figure 7.

The model is based on arguments which complete those for the simulation in Figure 2, and is of dynamic character for two reasons. Firstly, hydrogen peroxide, a product of the reaction, deactivates the GOD; moreover, catalase decomposes hydrogen peroxide, at the same time as it contributes to deactivate catalase. Accordingly, the presence of catalase causes the following sequence of phenomena: a) favours the action of GOD: b) increases the rate of hydrogen peroxide production: c) increases the rate of catalase deactivation: d) contributes to the decrease in GOD activity. The situation, therefore, can be described from the instantaneous rates of formation ( $v)$ and decomposition $(u)$ of the species involved, and thereafter their concentrations remaining after a time interval $\Delta t$. In other words:

1: Gluconic acid $(\mathrm{GnH})$ : where $S$ is the concentration of substrate (glucose), $m$ a Michaelian function, with or without inhibition, and where subscript $t$ refers to time: 


$$
\begin{aligned}
& v G n H_{t}=m\left(S_{t} ; G O D_{t}\right) \\
& G n H_{t}=G n H_{t-1}+v G n H_{t-1} \Delta t
\end{aligned}
$$

2: Hydrogen peroxide $(\mathrm{OX})$ : as a co-product of the reaction, its formation rate (in molar units) is given by the same equation as for gluconic acid:

$$
\begin{aligned}
& v O X_{t}=v G n H_{t} \\
& O X_{t}=G n H_{t}
\end{aligned}
$$

Hydrogen peroxide decomposition can be considered the result of two processes, one spontaneous, for which first order kinetics can be assumed as the simplest hypothesis:

$$
u_{1} O X_{t}=k_{1} O X_{t}=k_{1} G n H_{t}
$$

and another catalysed by catalase (CAT) and, therefore, Michaelian:

$$
u_{2} O X_{t}=m\left(O X_{t} ; C A T_{t}\right)=m\left(G n H_{t} ; C A T_{t}\right)
$$

In view of the fact that both processes, along with the formation of $O X$, are simply additive:

$$
\begin{aligned}
& u O X_{t}=u_{1} O X_{t}+u_{2} O X_{t} \\
& O X_{t}=O X_{t-1}+\Delta t\left(v O X_{t-1}-u O X_{t-1}\right)
\end{aligned}
$$

3: Glucose oxidase (GOD): is assumed to be affected by three types of processes:

3.1: spontaneous decomposition, with first order kinetics as the simplest hypothesis: 


$$
u_{1} G O D_{t}=g_{1} G O D_{t}
$$

3.2: deactivation by hydrogen peroxide, with second order kinetics, first in each species, again as the simplest hypothesis:

$$
u_{2} G O D_{t}=g_{2} O X_{t} G O D_{t}
$$

3.3: deactivation by the difference $(H)$ between the $\mathrm{pH}$ of maximum stability and the system $\mathrm{pH}$ :

$$
u_{3} G O D_{t}=g_{3} H_{t} G O D_{t}
$$

Summing the three rates:

$$
\begin{aligned}
& u G O D_{t}=G O D\left(g_{1}+g_{2} O X+g_{3} H_{t}\right) \\
& G O D_{t}=G O D_{t-1}-u G O D_{t-1} \Delta t
\end{aligned}
$$

With these principals, the rate of formation of gluconic acid in a time instant $t$, equivalent to the formation of hydrogen peroxide during the same instant, is modified in the following time instant, $t+1$, in agreement with the Michaelis-Menten equation. This arises in virtue of the variation in substrate concentration and also by the fall in the concentration of GOD during the time period $\Delta t$.

Accordingly, numerical integration of the net rate equations (16a) and (23a) with respect to time, allows to calculate the concentration of product and enzyme activity. Thereafter minimizing the quadratic differences between these and the experimental values, it is possible to estimate the parameters involved in (16a) to (23b). The solution obtained (Figure 7) presents a 
good fit to the experimental results (correlation coefficients of 0.978 and 0.995 ). The $\mathrm{pH}$ of maximum stability (5.49) agrees with the literature data and the data confirmed by direct measurement. The kinetic parameters, including the corresponding inhibitory effects, coincide with the results described in the previous section (the non-competitive inhibition does not appear less important).

Finally, attention should be drawn to a problem which is no easy to solve in the context of the system studied here and already discussed in a precedent work [29]. Recent estimates [33] of the kinetic parameters of the purified catalase from A. niger provides values of $322 \mathrm{mM} \mathrm{H}_{2} \mathrm{O}_{2}$ and $3.62 \times 10^{5} \mathrm{~mol} \mathrm{O}_{2}$ heme $\mathrm{e}^{-1} \mathrm{~s}^{-1}$ respectively for $K_{m}$ and $V_{m}$. But the conditions studied here are those of a more complex system, that does not necessarily guarantee a behavior of the catalase that allows the rigorous calculation of its kinetic parameters. Moreover, since catalase is an enzyme with a high conversion rate, even a very low concentration is enough to reduce the concentration of hydrogen peroxide in the system to values that are difficult to quantify with a reasonable degree of error (i.e. the rapid disappearance of the peroxide is guaranteed starting from a value of the maximum rate that can be inferior to the real one).

\section{CONCLUSIONS}

Oxidation of glucose catalysed by GOD can only be satisfactorily adjusted to a Michaelian model if diverse inhibitory effects of the substrate and products (gluconic acid and hydrogen peroxide), as well as possible interferences due to the catalase (often present in partially purified preparations of GOD), are taken into account. Many kinetically focused studies concentrate on the behaviour of the immobilized enzyme, applied to the development of glucose probes. Due to the very low substrate concentrations used in these studies, detection of the above-mentioned effects becomes difficult. In the microbiological field, on the contrary, the usual conditions involve higher concentrations of glucose, but the systems studied contain many components that 
vary with time, hindering the kinetic approach (it should be pointed out, however, that the indications of inhibitory effects originate basically from this area).

In this work, instigated by previous observations in microbial cultures, the complexity of these systems is simplified without reducing their habitual glucose levels, and maintaining the presence of catalase. In this way, it was possible to show that the kinetics of the GOD is affected by: 1) Substrate inhibition, 2) Competitive inhibition by gluconic acid, 3) A decrease of the reaction rate due to diffusional restrictions determined by the viscosity of the gluconic acid, 4) A decrease of the reaction rate due to enzyme deactivation by hydrogen peroxide, a feature (with some phenomenological resemblance to true substrate inhibition) which disappears when catalase is present. Working within a wide interval of glucose concentrations, or in the context of microbial cultures for production of GOD, the above effects must necessarily be considered. The models proposed in this paper allow us to evaluate them.

\section{REFERENCES}

[1] Nicol MJ, Duke FR. Substrate inhibition with glucose oxidase. J Biol Chem 1966;241(18):4292-93.

[2] Barker SA, Shirley JA. Glucose Oxidase. In: Rose AH, editor. Microbial enzymes and bioconversions. Economic Microbiology, vol. 5. London: Academic Press, 1980. p. 171-82.

[3] Tse PHS, Gough DA. Time-dependent inactivation of immobilized glucose oxidase and catalase. Biotechnol Bioeng 1987;29:705-13.

[4] Kozhukharova A., Kirova N, Popova Y, Batsalova K. Properties of glucose oxidase inmobilized in gel of polyvinylalcohol. Biotechnol Bioeng 1988;32:245-48.

[5] Bright HJ, Gibson QH. The oxidation of 1-deuterated glucose by glucose oxidase. J Biol Chem 1967; 242(5):994-1003.

[6] Nakamura S, Ogura Y. Action mechanism of glucose oxidase of Aspergillus niger. J Biochem Tokyo 1968;63(3):308-16.

[7] Weetall HH, Hersh LS. (1970). Preparation and characterization of glucose oxidase covalently linked to nickel oxide. Biochim Biophys Acta 1970;206:54-60.

[8] Bourdillon C, Hervagault C, Thomas D. Increase in operational stability of immobilized glucose oxidase by the use of an artificial cosubstrate. Biotechnol Bioeng 1985;27:1619-22.

[9] Demura M, Asakura T. Immobilization of glucose oxidase with Bombyx mori silkfibroin by only stretching treatment and its application to glucose sensor. Biotechnol Bioeng 1989;33:598-603.

[10] Hayashi S, Nakamura S. Comparison of fungal glucose oxidases. Chemical, physicochemical and immunological studies. Biochim Biophys Acta 1976;438:37-48.

[11] Bouin JC, Atallah MT, Hultin HO. The glucose oxidase-catalase system. In: Wood WA, editor. Methods in Enzymology, vol. 44. New York: Academic Press, 1977. p. 478-88.

[12] Pieters BR, Bardeletti G. Enzyme immobilization on a low-cost magnetic support: Kinetic studies on immobilized and coimmobilized glucose oxidase and glucoamylase. Enzyme Microb Technol 1992;14:361-70. 
[13] Witteveen CFB, Van De Vondervoort PJI, Van Den Broeck CH, Van Engelenburg FAC, De Graaff LH, Hillebrand MHBC, Schaap PJ, Visser J. Induction of glucose oxidase, catalase, and lactonase in Aspergillus niger. Curr Genet 1993;24:408-16.

[14] Van Stroe-Biezen SAM, Van Der Loo JMH, Janssen LJJ, Everaerts FM. Determination of the inherent kinetics of immobilized glucose oxidase using a diffusion cell. Bioprocess Eng 1996;15(2):87-94.

[15] Swoboda BEP, Massey V. Purification and properties of the glucose oxidase from Aspergillus niger. J Biol Chem 1965;240(5):2209-15.

[16] Miura Y, Tsuchiya K, Tsusho H, Miyamoto K. Kinetic studies of gluconic acid fermentation, using Aspergillus niger. J Ferment Technol 1970;48(12):795-803.

[17] Kuzuhara A., Asakura T, Tomoda R, Matsunaga T. Use of silk fibroin for enzyme membrane. J Biotechnol 1987;5:199-207.

[18] Takegawa K, Fujiwara K, Iwahara S, Yamamoto K, Tochikura T. Effect of deglycosylation of Nlinked sugar chains on glucose oxidase from Aspergillus niger. Biochem Cell Biol 1989;67:460-64.

[19] Wolowacz SE, Yon Hin BFY, Lowe CR. Covalent electropolymerization of glucose oxidase in polypyrrole. Anal Chem 1992;64:1541-45.

[20] Nakamura T, Ogura Y. Kinetic studies on the action of glucose oxidase. J Biochem Tokyo 1962;52(3):214-20.

[21] Rogers MJ, Brandt KG. Interaction of D-glucal with Aspergilllus niger glucose oxidase. Biochemistry 1971;10(25):4624-30.

[22] Velizarov S, Beschkov V. Biotransformation of glucose to free gluconic acid by Gluconobacter oxydans: Substrate and product inhibition situations. Proc Biochem 1998;33(5):527-34.

[23] Crueger A, Crueger W. Glucose transforming enzymes. In: Fogarty, WM, Kelly CT, editors. Microbial enzymes and biotechnology, New York: Elsevier Applied Science, 1990. p. 177-226.

[24] Tse PHS, Leypoldt JK, Gough DA Determination of the intrinsic kinetic constants of immobilized glucose oxidase and catalase. Biotechnol Bioeng 1987;29:696-704.

[25] Nakamura S, Fujiki S. Comparative studies on the glucose oxidases of Aspergillus niger and Penicillium amagasakiense. J Biochem Tokyo 1968;63(1):51-58.

[26] Hayashi S, Nakamura S. Multiple forms of glucose oxidase with different carbohydrate compositions. Biochim Biophys Acta 1981;657:40-51.

[27] Mirón J, González MP, Pastrana L, Murado MA. Diauxic production of glucose oxidase by Aspergillus niger in submerged culture. A dynamic model. Enzyme Microb Technol 2002;31:61520.

[28] Lloyd JB, Whelan WJ. An improved method for enzymic determination of glucose in the presence of maltose. J Anal Biochem 1969;30:467-70.

[29] Fiedurek J, Rogalski J, Ilczuk Z, Leonowcz A. Screening and mutagenesis of moulds for the improvement of glucose oxidase production. Enzyme Microb Technol 1986;8:734-6.

[30] Lee EYC, Carter JH, Nielsen LD, Fisher EH. Purification and properties of yeast amylo-1,6glucosidase-oligo-1,4 1,4-glucantransferase. Biochemistry 1970;9(11):2347-55.

[31] Möllering H, Bergmeyer HU. (1974). D-Gluconate. In: Bergmeyer HU, editor. Methods of Enzymatic Analysis, vol 3. New York: Academic Press, 1974. p. 1243-47.

[32] Tan Y, Wang Z-X, Marshall KC. Modeling substrate inhibition of microbial growth. Biotechnol Bioeng 1996;52:602-08.

[33] Miranda M, Murado MA, Sanromán A, Lema JM. (1991). Mass transfer control of enzymatic hydrolysis of polysaccharides by glucoamylase. Enzyme Microb Technol 1991;13:142-47.

[34] Sanromán A., Murado MA, Lema JM. (1996). The influence of substrate structure on the kinetics of the hydrolysis of starch by glucoamylase. Appl Biochem Biotechnol 1996;59:329-36.

[35] Pastrana LM, González MaP, Mirón J, Murado MA. A new device for measuring diffusional restrictions and modelling subtrate inhibition in a starch-glucoamylase system. Biotechnol Lett 1998;20(2):127-30. 


\begin{tabular}{|c|c|}
\hline \multicolumn{2}{|c|}{ Abbreviations and symbolic notations used } \\
\hline GOD & glucose oxidase \\
\hline PO & peroxidase \\
\hline FAD & flavin adenine dinucleotide \\
\hline $\mathrm{NADP}^{+}\left(\right.$and $\left.\mathrm{NADPH}+\mathrm{H}^{+}\right)$ & nicotinamide adenine dinucleotide phosphate (and reduced form) \\
\hline ATP (and ADP) & adenosin triphosphate (and diphospate) \\
\hline $\mathrm{SI}$ & substrate inhibition \\
\hline \multicolumn{2}{|l|}{ Simulation } \\
\hline vGnH & rate of gluconate production \\
\hline VOX & rate of hydrogen peroxide production \\
\hline$S$ & substrate concentration \\
\hline$O X$ & hydrogen peroxide formed after a time interval $t$ \\
\hline$V_{m}$ & maximum rate of product formation (Michaelis and Menten model) \\
\hline$K_{m}$ & Michaelis constant \\
\hline UGOD & rate of GOD deactivation \\
\hline GOD & concentration of GOD (with GOD 0 as initial value) \\
\hline$g$ & kinetic parameter (second order) \\
\hline \multicolumn{2}{|l|}{ Michaelian } \\
\hline \multicolumn{2}{|l|}{ approaches } \\
\hline$K_{s}$ & substrate inhibition coefficient \\
\hline$I_{c}$ & concentration of competitive inhibitor \\
\hline$K_{c}$ & competitive inhibitor coefficient \\
\hline $\mathrm{la}$ & concentration of uncompetitive inhibitor \\
\hline$K_{a}$ & uncompetitive inhibition coefficient \\
\hline $\ln$ & concentration of non-competitive inhibitor \\
\hline$K_{n}$ & non-competitive inhibition coefficient \\
\hline \multicolumn{2}{|l|}{ Dynamic model } \\
\hline$v G n H_{t}$ & rate of gluconate production in a time instant $t$ \\
\hline$m$ & Michaelian function \\
\hline$S$ & substrate concentration \\
\hline$G O D_{t}$ & GOD concentration \\
\hline $\mathrm{GnH}_{\mathrm{t}}$ & gluconate formed after a time interval $t$ \\
\hline VOX & rate of hydrogen peroxide production in a time instant $t$ \\
\hline$O X_{t}$ & hydrogen peroxide formed after a time interval $t$ \\
\hline $\mathrm{u}_{1} \mathrm{OX} \mathrm{X}_{t}$ & rate of spontaneous (firt order) decomposition of hydrogen peroxide \\
\hline$k_{1}$ & kinetic parameter (first order) \\
\hline $\mathrm{u}_{2} \mathrm{O} X_{t}$ & rate of hydrogen peroxide decomposition by catalase (first order) \\
\hline$C A T_{t}$ & catalase concentration \\
\hline uOXt & rate (total) of hydrogen peroxide formation in a time instant $t$ \\
\hline$u_{1} G O D_{t}$ & rate of spontaneous (first order) decomposition of GOD \\
\hline$g_{1}$ & kinetic parameter (first order) \\
\hline $\mathrm{U}_{2} G O D_{t}$ & rate of GOD deactivation (second order) \\
\hline$g_{2}$ & kinetic parameter (second order) \\
\hline$u_{3} G O D_{t}$ & rate of GOD deactivation (second order) \\
\hline$g_{3}$ & kinetic parameter (second order) \\
\hline$H_{t}$ & difference between $\mathrm{pH}$ of maximum stability of GOD and real $\mathrm{pH}$ \\
\hline$u G O D_{t}$ & rate (total) of GOD decomposition \\
\hline
\end{tabular}


TABLE 1: Analytical details of the GOD preparations used in the kinetic experiments.

\begin{tabular}{lcc}
\hline & Raw preparation & SIGMA G-7141 \\
\cline { 2 - 3 } GOD (EU/mg solid) & 16.7 & 172.0 \\
GOD (EU/mg protein) & 34.79 & 236.52 \\
Catalase (EU/mg solid) & 7.93 & $\mathrm{tr}$ \\
Catalase (EU/mg protein) & 16.52 & $\mathrm{tr}$ \\
Catalase / GOD & 0.475 & $\mathrm{tr}$ \\
FAD (mg/mg solid) & 0.002 & 0.007 \\
Total carbohydrates (mg/mg solid) & 0.22 & 0.11 \\
Protein (mg/mg solid) & 0.48 & 0.73 \\
\hline
\end{tabular}

TABLE 2: Initial models applied to the analysis of the kinetic results. $V_{m}$ : maximum rate; $K_{m}$ : Michaelis-Menten constant; $S$ : substrate concentration. In all cases the factor $F_{i}$ takes the form $F_{i}=K_{i} I_{i}$, where $I_{i}$ is the inhibitor concentration and $K_{i}$ is the inhibition constant (dimensions $1 / I_{i}$ ). In substrate inhibition, $I_{i}=S$.
(1) without inhibition

$$
V=\frac{V_{m} S}{K_{m}+S}
$$
(2) substrate inhibition$$
V=\frac{V_{m} S}{K_{m}+S F_{s}}=\frac{V_{m} S}{K_{m}+S+K_{s} S^{2}}
$$
(3) competitive inhibition

$$
V=\frac{V_{m} S}{K_{m} F_{c}+S}
$$
(4) noncompetitive inhibition$$
V=\frac{V_{m} S}{\left(K_{m}+S\right) F_{n}}
$$
(5) uncompetitive inhibition $\quad V=\frac{V_{m} S}{K_{m}+S F_{a}}$ 
TABLE 3: Parameters for models (1) and (2), applied to the experimental results of figure 1.

\begin{tabular}{lccc}
\hline & Without substrate inhibition & & With substrate inhibition \\
$\mathrm{K}_{m}\left(g . \mathrm{L}^{-1}\right)$ & 2.613 & & 3.523 \\
$\mathrm{~V}_{\mathrm{m}}\left(\mathrm{mM} \cdot \mathrm{min}^{-1}\right)$ & 8.052 & & 9.003 \\
$\mathrm{~K}_{\mathrm{s}}(1 /[\mathrm{S}])$ & - & & 0.0010 \\
Correlation $(r)$ observed $v$. expected values & 0.974 & 0.996 \\
\hline
\end{tabular}

TABLE 4: Parameters and fits of the specified models for the kinetic description of GOD.

\begin{tabular}{|c|c|c|c|c|}
\hline & $(2)$ & (12) & (13) & (14) \\
\hline$K_{m}\left(g \cdot L^{-1}\right)$ & 3.523 & 5.888 & 5.708 & 4.364 \\
\hline $\mathrm{V}_{\mathrm{m}}\left(\mathrm{mM} \cdot \mathrm{min}^{-1}\right)$ & 9.003 & 11.091 & 9.526 & 9.350 \\
\hline $\mathrm{K}_{\mathrm{s}}\left(\mathrm{L} . \mathrm{g}^{-1}\right)$ & 0.0010 & 0.0016 & 0.0015 & 0.0017 \\
\hline $\mathrm{K}_{\mathrm{c}}\left(\mathrm{L} . \mathrm{g}^{-1}\right)$ & - & - & - & 0.015 \\
\hline$K_{n}\left(L . g^{-1}\right)$ & - & 0.014 & 0.00015 & 0.00011 \\
\hline$r$ (observed $v$. expected) & 0.996 & 0.982 & 0.993 & 0.997 \\
\hline
\end{tabular}




\section{FIGURE CAPTIONS}

Figure 1: Kinetics of the preparations of GOD described in Table 1 (left: impure preparation; right: SIGMA product). S: substrate concentration; $V$ : rate of production of gluconic acid. The experimental results (points) show fits to the Michaelis-Menten model, without (dotted line) and with (continuous line) substrate inhibition.

Figure 2: Simulation of the interference by hydrogen peroxide in the kinetic profiles obtained with increasing reaction periods. Simulation was performed using the equations (6) to (9) with the following parameters: $V_{\mathrm{m}}=0.8 ; K_{\mathrm{m}}=2 ; G O D_{(\mathrm{t}=0)}=1 ; S_{0}=100 ; g=0.005 ; t_{1}=75 ; t_{2}=100 ; t_{3}=110$. Left: expected results (points) and their fit (lines) to equation (2) of Table 2 (note the similarity of cases $t_{2}$ and $t_{3}$ with the true substrate inhibition). Right: residuals of the corresponding fits (note the pronounced autocorrelation).

Figure 3: Production of gluconic acid and GOD remaining in the impure preparation (left) and the SIGMA product (right), without (white symbols) and with (black symbols) a supplement of $100 \mathrm{EU} / \mathrm{mL}$ catalase. The solution was buffered with $0.2 \mathrm{M}$ citrate-phosphate; $\mathrm{pH}=5.1$. Initial GOD: $2 \mathrm{EU} / \mathrm{mL}$; Initial substrate: $25 \mathrm{mg} / \mathrm{mL}$.

Figure 4: Kinetics of the impure preparation of GOD, adjusted to the Michaelis-Menten model without (fine line) and with (thick line) SI. The bars indicate the confidence interval ( $\mathrm{n}=3$; $\alpha<0.05$ ) of the production slopes of gluconic acid, calculated from five intervals of maximum length of 4 minutes.

Figure 5: Kinetics of GOD in absence ( $\bullet$ ) and presence of gluconic acid $(\nabla, \diamond, \bigcirc$ and $\square$ : $12.75 ; 25.49 ; 50.98$ and $101.96 \mathrm{mM}$, respectively), fitted (continuous lines) to model (14).

Figure 6: Correlation between expected and observed results (left) and residual distributions (right) as a function of the concentration of gluconic acid (right), using equations (12), (13) and (14): A, B and C respectively.

Figure 7: Gluconic acid produced (left) and remaining GOD (right), in incubations of the impure preparation, without $(\bigcirc)$ and with catalase supplement $(\bullet)$, under the conditions described in 
figure 3. The continuous lines represent the corresponding fits to the model proposed in equations (15a) to (23b).

\section{FIGURE 1}

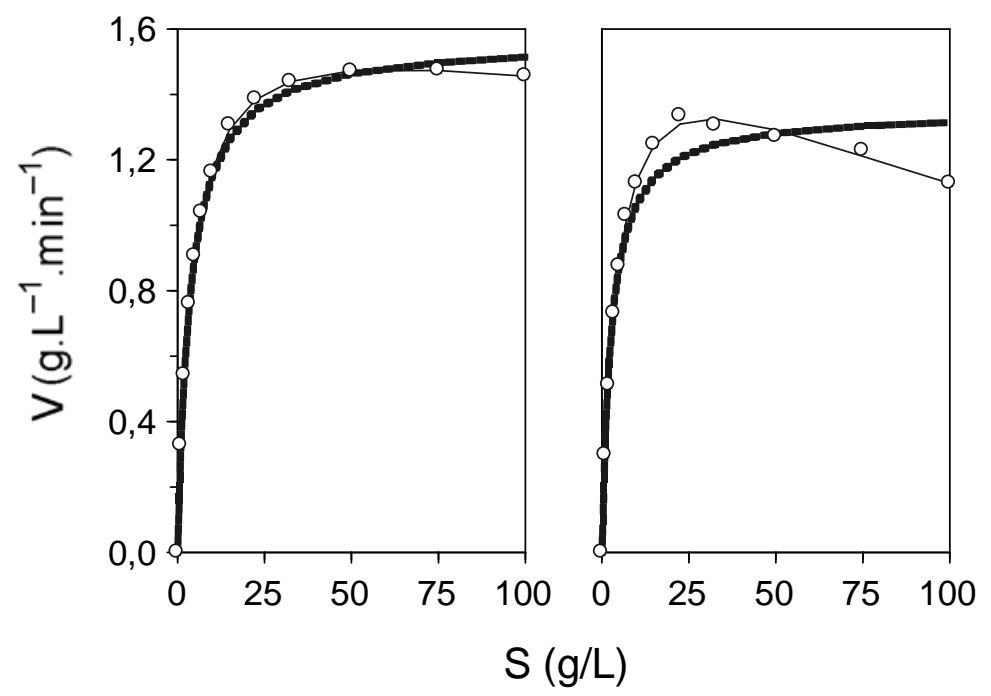


FIGURE 2
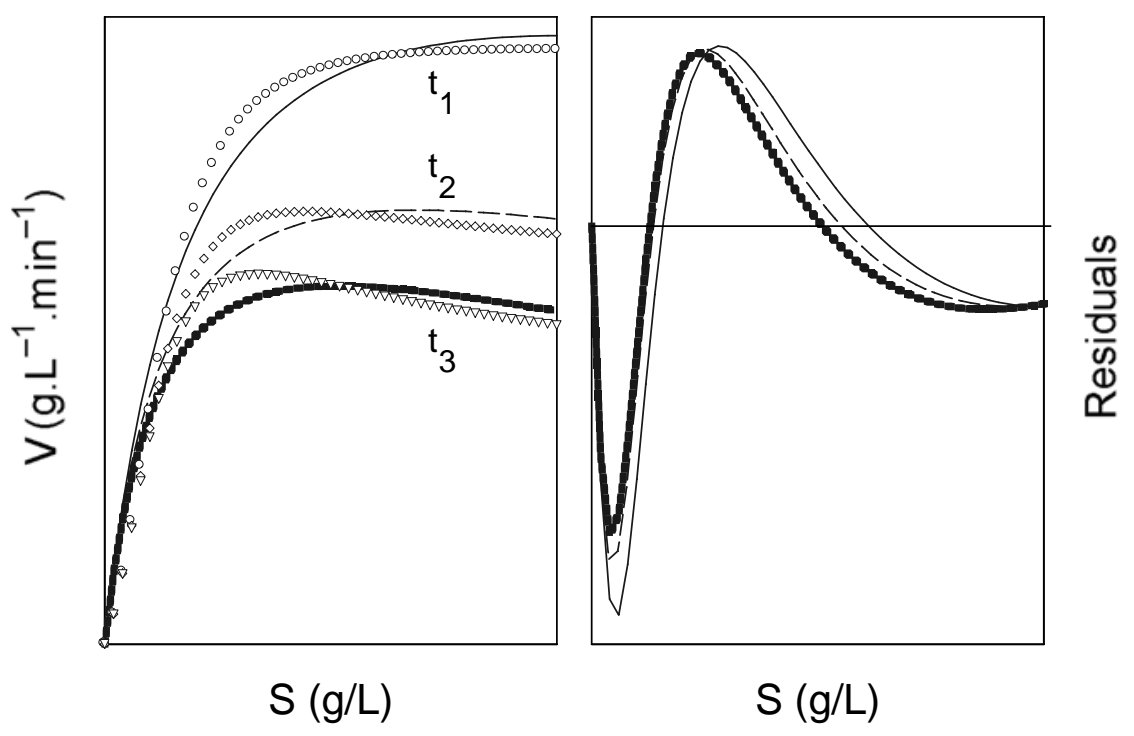
FIGURE 3

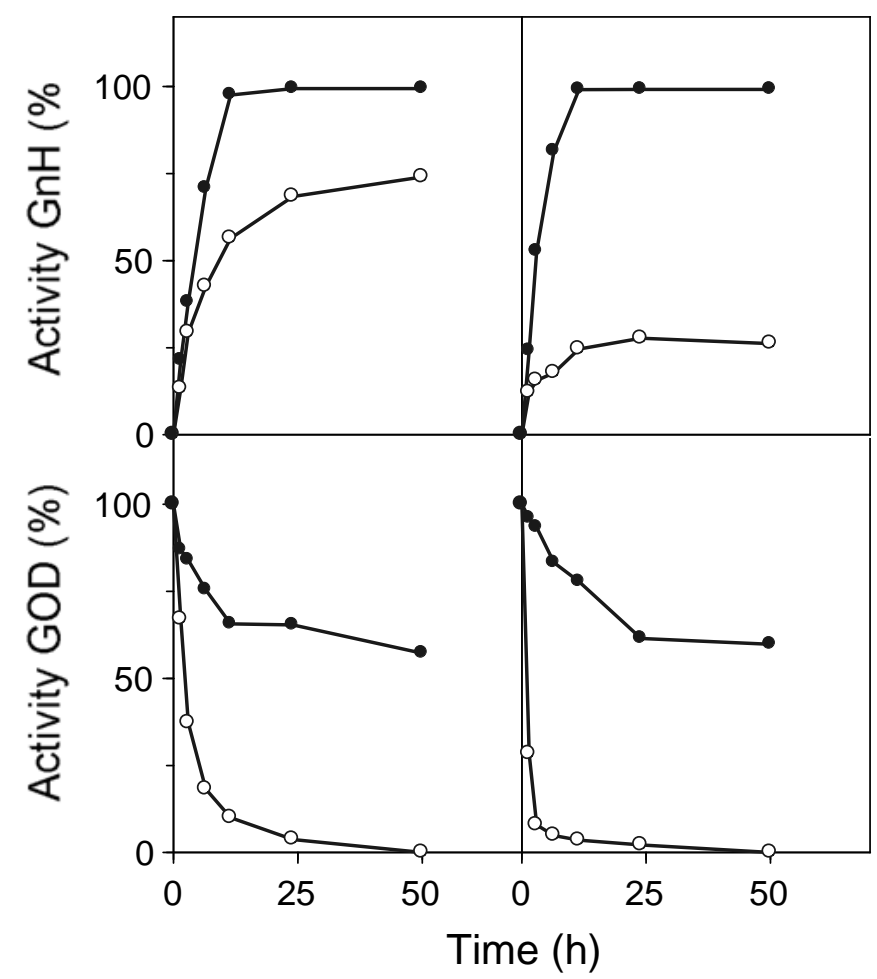


FIGURE 4

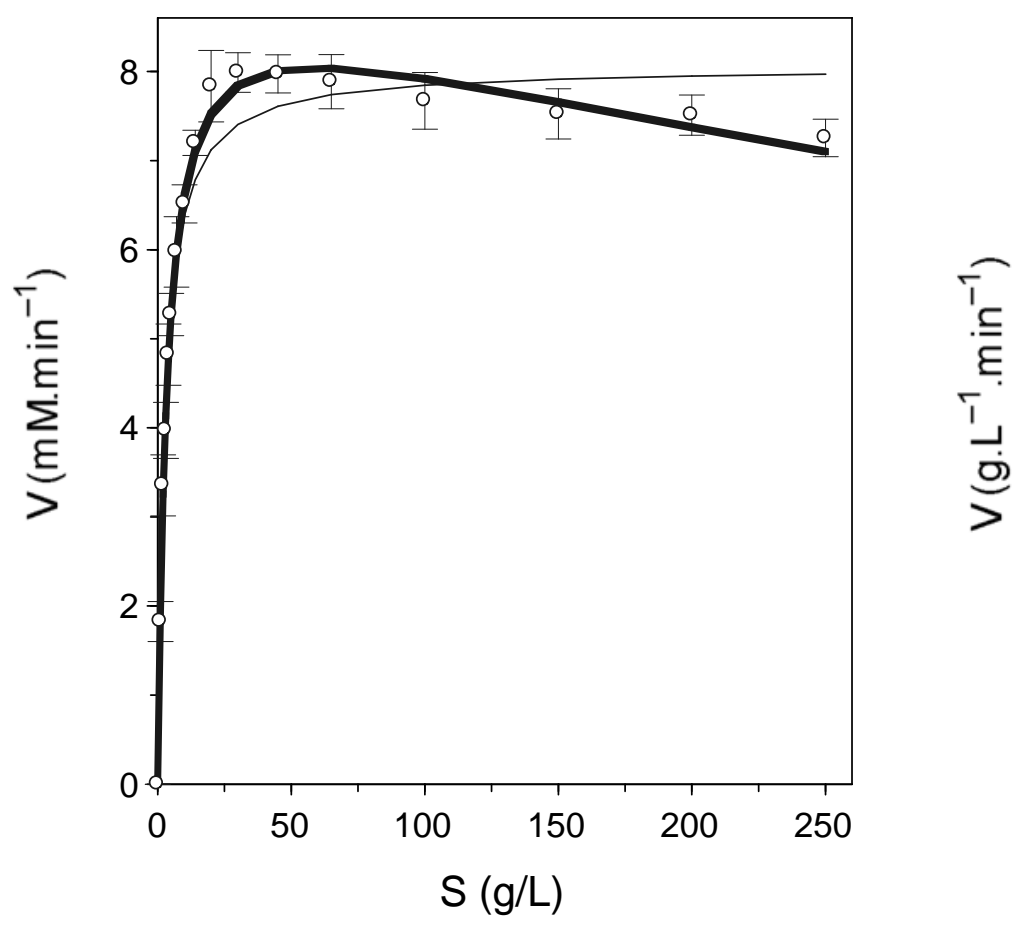

$-26-$ 
FIGURE 5

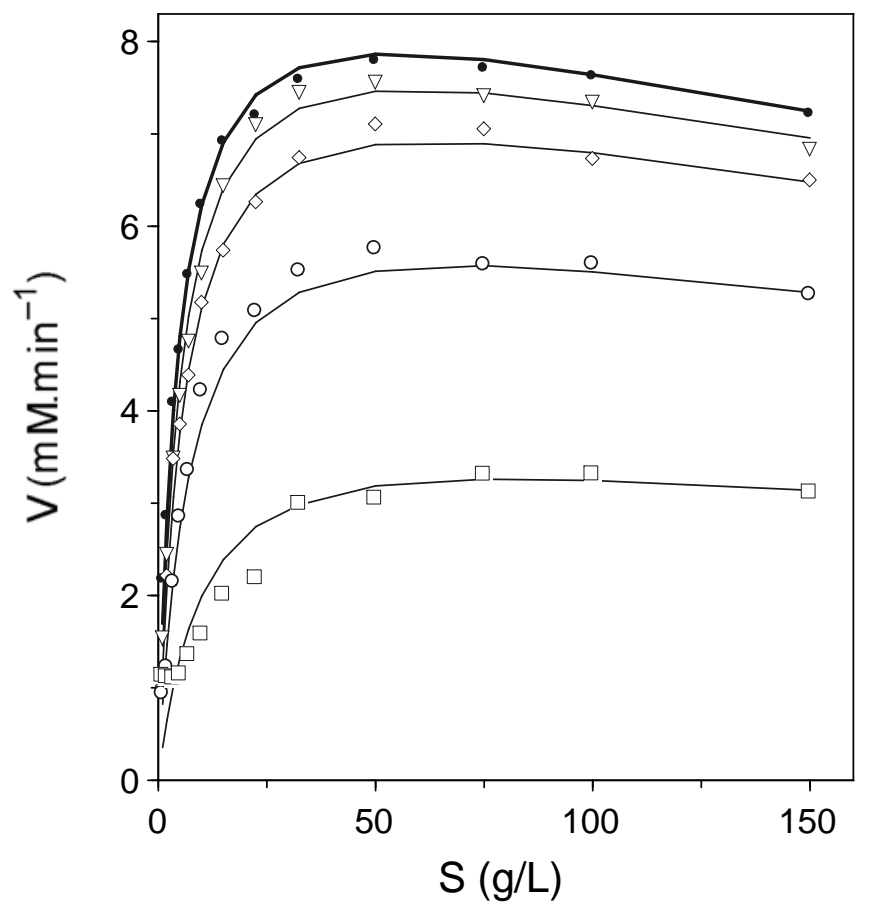


FIGURE 6
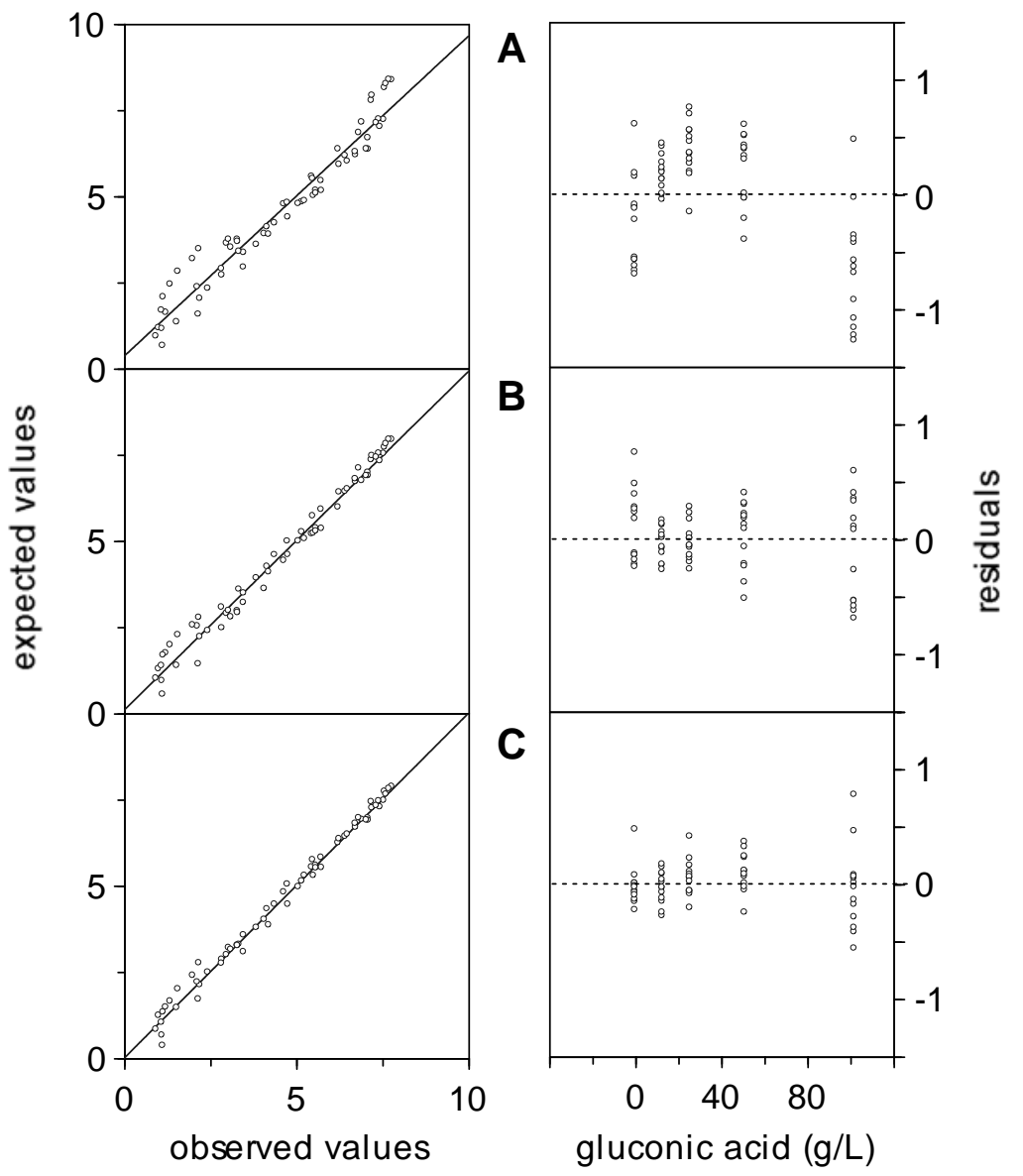
FIGURE 7

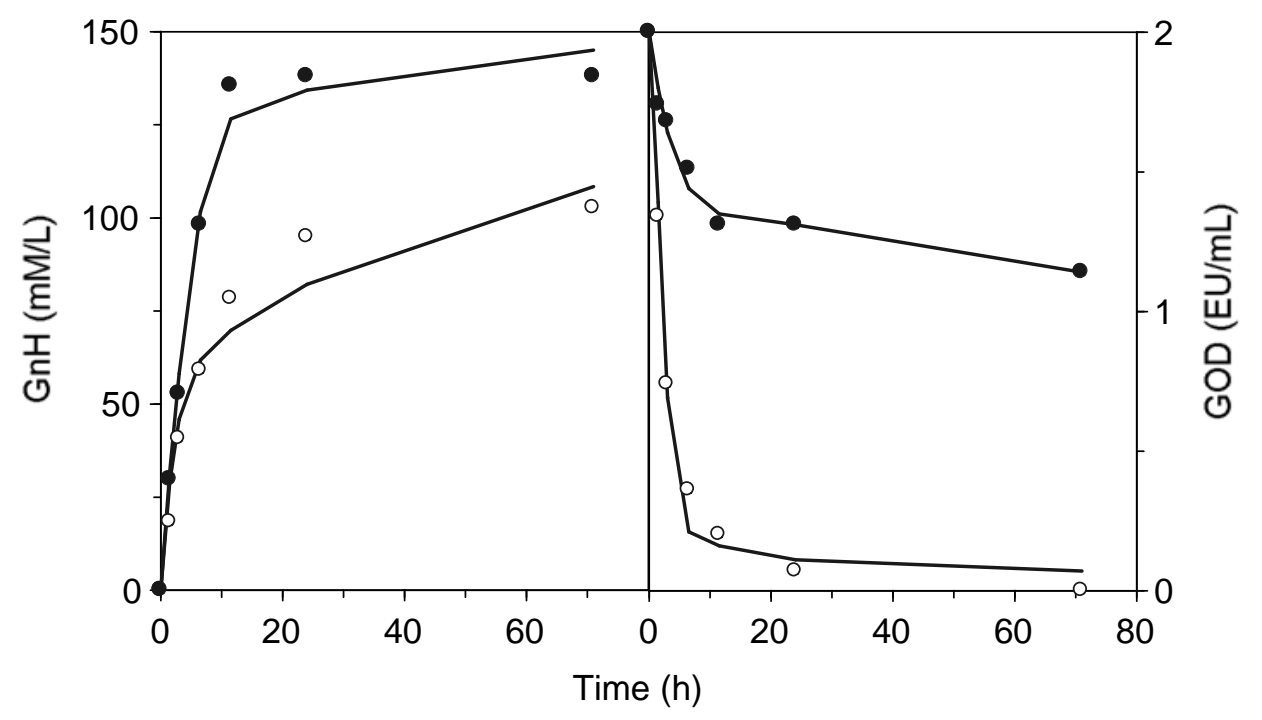

